\title{
Confiança, atitudes, informação: um estudo sobre a percepção da pandemia de COVID-19 em 12 cidades brasileiras
}

\author{
Trust, attitudes, information: a study on the perception \\ of the COVID-19 pandemic in 12 Brazilian cities
}

Luisa Massarani (https://orcid.org/0000-0002-5710-7242) ${ }^{1}$

Ione Maria Mendes (https://orcid.org/0000-0001-9993-8039) ${ }^{2}$

Vanessa Fagundes (https://orcid.org/0000-0002-8018-8490) ${ }^{3}$

Carmelo Polino (https://orcid.org/0000-0003-1789-8024) ${ }^{4}$

Yurij Castelfranchi (https://orcid.org/0000-0003-4003-5956) ${ }^{5}$

Bertha Maakaroun (https://orcid.org/0000-0003-2713-1708) ${ }^{6}$

${ }^{1}$ Instituto Nacional de Comunicação Pública da Ciência e Tecnologia, Centro de Documentação e História da Saúde, Casa de Oswaldo Cruz, Fundação Oswaldo Cruz. Av. Brasil 4365, Manguinhos. 21040 900 Rio de Janeiro RJ Brasil. luisa.massarani@ fiocruz.br

${ }^{2}$ Programa de PósGraduação em Saúde Global e Sustentabilidade, Faculdade de Saúde Pública, Universidade de São Paulo.

São Paulo SP Brasil.

${ }^{3}$ Programa de Pós-

Graduação em Sociologia, Universidade Federal de

Minas Gerais (UFMG). Belo

Horizonte MG Brasil.

${ }^{4}$ Departamento de Filosofía,

Universidad de Oviedo.

Oviedo España.

${ }^{5}$ Departamento de

Sociologia, UFMG. Belo

Horizonte MG Brasil.

${ }^{6}$ Universidade Estadual

do Rio de Janeiro. Rio de

Janeiro RJ Brasil.

\begin{abstract}
In this study, the perception of Brazilians regarding COVID-19 in 12 cities in the country was analyzed. Issues about the severity and dangers of the disease, sources of information and reliability, checking information, attitudes, precautions, and priorities for coping and trusting relationships in science were addressed. This study was carried out in the context of broader research on how Brazilians perceive the Oswaldo Cruz Foundation (Fiocruz). The survey was applied between May 18 and June 10, 2020, with 1,643 residents over 16 years of age in Brazilian cities where Fiocruz has a technical unit or office. The results reveal that the majority of the interviewees appreciate the seriousness of the pandemic, the importance of being properly informed, considers the measures indicated by health authorities important and trusts scientists and scientific institutions as sources of information. With the sense of urgency and experience of uncertainties about the present and the future, the need for strengthening of trust in institutions is present.
\end{abstract}

Key words COVID-19, SARS-CoV-2, Pandemic, Public perception
Resumo Neste estudo, analisamos a percepção de brasileiros e brasileiras sobre a COVID-19 em 12 cidades do país. Foram abordadas questões sobre a gravidade e os perigos da doença, fontes de informações e confiabilidade, checagem de informações, atitudes, precauções e prioridades para o enfrentamento e relações de confiança na ciência. Este estudo foi realizado no contexto de uma pesquisa mais ampla sobre como brasileiros e brasileiras veem a Fundação Oswaldo Cruz (Fiocruz). A survey foi aplicada no período de 18 de maio e 10 de junho de $2020 \mathrm{com} 1.643$ moradores com mais de 16 anos nas cidades brasileiras em que a Fiocruz possui unidade ou escritório técnico. Os resultados revelam que boa parte dos entrevistados reconhece a gravidade da pandemia, a importância de se informar corretamente, considera as medidas indicadas por autoridades de saúde válidas e confia em cientistas e instituições científicas como fontes de informação. Com o senso de urgência e a potencialização e vivência das incertezas acerca do presente e do futuro, ofortalecimento da confiança nas instituições se faz presente.

Palavras-chave COVID-19, Sars-CoV-2, Pandemia, Percepção pública 


\section{Introdução}

Em dezembro de 2019, a Organização Mundial da Saúde (OMS) foi notificada de vários casos de pneumonia de origem indeterminada na cidade chinesa de Wuhan. Uma semana depois, em 7 de janeiro de 2020, as autoridades chinesas identificaram a origem das infecções: um novo tipo de coronavírus, o Sars-CoV-2, causador da COVID-19. Por conta da rápida propagação do vírus em diversos países e dos altos números de mortes, em 11 de março, a OMS declarou se tratar de uma pandemia e recomendou as primeiras medidas de enfrentamento que foram adotadas ou não em diferentes países e regiões de acordo com possibilidades socioeconômicas, políticas, culturais, modos de vida e estruturação dos serviços de saúde disponíveis.

As consequências dessa pandemia são muitas e ainda estão em andamento, uma vez que está longe de ser mitigada e sob controle. São consequências em diferentes níveis que afetam não só a saúde, mas também a economia, o emprego, a educação e as relações sociais, realçando as assimetrias e desigualdades. Segundo Morens et al. ${ }^{1}$, no futuro próximo, ocorrerão outras doenças que apresentarão desafios consideráveis, com destaque para pandemias com altas taxas de mortalidade. Uma das áreas mais negligenciadas de risco emergente está diretamente relacionada com as mudanças recentes na economia política internacional, com a aceleração e evolução de novos patógenos, limitando o alcance e a eficácia de tecnologias estabelecidas de prevenção de saúde pública, além de crises políticas e sociais, aumento da criminalidade e de conflitos violentos ${ }^{2}$.

Neste contexto, os processos de interdependência planetária, quebra de barreiras espaciais e crescente migração e circulação de pessoas ao redor do mundo configuraram um terreno fértil para o descontrole da disseminação e da mitigação do contágio da COVID-19. Os impactos foram sentidos em diversas áreas, mesmo que de forma desigual, dependendo das condições socioeconômicas, políticas e culturais de cada país e de suas diferentes regiões ${ }^{3-5}$. Como exemplo, no Brasil, comparando brancos, pardos e negros que foram hospitalizados, os negros apresentaram risco significativamente maior de mortalidade. Na região Norte do país, os pacientes hospitalizados apresentam maior risco de morte por COVID-19 do que na região Centro-Sul ${ }^{5}$.

A gestão de uma pandemia decorrente de um vírus respiratório, segundo Garcia et al. ${ }^{6}$, requer capacidade de saúde pública para resposta co- ordenada para implementação de restrições de circulação obrigatórias (bloqueios, quarentenas, toque de recolher) e voluntárias (distanciamento social e uso de máscaras), testes em grande escala para rastreamento dos infectados, vigilância rigorosa e monitoramento em tempo real da trajetória da epidemia, além de gestão de recursos humanos para cuidados de saúde e serviços essenciais. As medidas restritivas, como o confinamento, são especialmente desafiadoras em algumas localidades. Com a desigualdade social entre países e no interior de cada país, a falta de equipamentos e estrutura adequados para o enfrentamento da doença, sistemas de saúde pouco estruturados ou em diferentes níveis de estruturação, a escassez de vacina, a falta de tratamento específico para a doença, a escassez de testes e insumos, a contaminação progressiva e o risco de agravamento da doença que pode levar a muitas mortes, a COVID-19 coloca o mundo diante de um grande desafio. Para superá-lo, torna-se crucial o engajamento da população, muitas vezes voluntário, às recomendações da OMS e de autoridades sanitárias locais sobre distanciamento social, isolamento, uso de máscaras e, em breve, sobre a vacinação.

O sucesso de políticas públicas para evitar o contágio e barrar a transmissão da doença depende, em parte, da percepção sobre ciência e cientistas, do grau de confiança nas instituições e na mídia e na percepção de risco das pessoas sobre a pandemia. Tal percepção apresenta variações em diferentes grupos por ser socialmente construída, determinada por fatores socioeconômicos, políticos e culturais e afetada pelos efeitos da comunicação e informação sobre riscos com seus diferentes enquadramentos ${ }^{7}$. A comunicação de risco torna-se, assim, relevante para a gestão de situações como a imposta pela pandemia de COVID-19.

Entendida também como a "troca de informação, aconselhamento e opiniões em tempo real entre peritos, líderes comunitários, funcionários e as pessoas que estão em risco"s, a comunicação de risco é parte integrante de qualquer resposta de emergência, permitindo às pessoas compreender e adotar comportamentos de proteção, além de propiciar às autoridades o processo de escuta das populações e de responder às suas demandas com aconselhamento relevante e confiável. Essa é uma arena complexa. Além de lidar com incertezas inerentes à ciência, reúne atores diversos, tais como cientistas, pesquisadores, profissionais de saúde, gestores, políticos, meios de comunicação e redes sociais, cujas narrativas são, muitas 
vezes, divergentes e atendem a interesses específicos $^{9-11}$. Também cabe destacar a importância da informação em saúde que fornece subsídios para o próprio setor saúde e serve "tanto à produção de informações importantes e pertinentes quanto, também, à disseminação de equívocos e de produtos de manipulação indevida dos dados em saúde" ${ }^{12}$.

As redes sociais digitais, por sua vez, adicionam um novo desafio relacionado à desinformação, já que fazem circular discursos não embasados em evidências científicas e dão voz a opiniões que podem confundir ainda mais o entendimento real dos riscos por parte da população exposta.

As fake news, ou notícias falsas, são agentes de desinformação. Deliberadamente criadas e disseminadas com o intuito de causar prejuízos e influenciar pessoas ${ }^{13}$, as fake news circulam especialmente no ambiente online, sobretudo nas redes sociais digitais, e complexificam os processos de comunicação (e de comunicação de risco) ao gerar insegurança com relação aos dados e às fontes. Conhecer as fontes que inspiram mais confiança e as novas rotinas associadas ao consumo de informação, como a checagem, contribuem para entender os impactos da desinformação na percepção de risco.

Nesse sentido, estudos de percepção e seus impactos são ferramentas importantes para entender como os indivíduos respondem aos riscos em diferentes contextos sociais e, principalmente, como fornecem possibilidades de colaboração para a construção de pontes de conexão adequadas e de comunicação, identificando fatores determinantes da aceitação e adoção de medidas de proteção:

Em geral, estudos sobre percepção buscam compreender como os indivíduos respondem ao risco $e$ às informações disponíveis sobre esse risco, que fatores interferem nas suas respostas, como respondem às dimensões sociais (implícitas ou não) nas suas situações de vida real, como definem os riscos, como se sentem atingidos por esses riscos e como imaginam enfrentá-los ${ }^{7}$ (p.1223).

Desde que a OMS declarou "Emergência de Saúde Pública de Importância Internacional" (ESPII) devido a rápida disseminação do contágio da COVID-19 somada ao alto risco de agravamento da doença ${ }^{14}$, estão sendo realizados vários estudos nacionais e internacionais de percepção e entendimento da doença por parte da população com diferentes abordagens, metodologias e datas de coleta. Na Alemanha, por exemplo, em coleta realizada entre os dias 19 e 23 de março de 2020 entre adultos que não testaram positi- vo para COVID-19, 22,7\% acreditavam que seriam infectados e 33,7\% acreditavam que alguma pessoa da família ou amigos seriam infectados pelo Sars-CoV-2 em um futuro próximo. Além disso, $94,6 \%$ se sentiam protegidos em suas casas, $38,4 \%$ na casa de outras pessoas, poucos se sentiam seguros em shoppings e/ou restaurantes $(8,3 \%)$ e apenas $7,3 \% \%$ em espaços ou transporte público. Quanto às medidas de precaução, $87,2 \%$ afirmaram lavar ou desinfetar as mãos com frequência maior que a usual, $87,2 \%$ estavam evitando eventos e espaços públicos, $85,2 \%$ evitavam transporte público e 78,0\%, o contato com pessoas consideradas do grupo de risco ${ }^{15}$.

Uma pesquisa comparativa, coordenada pelo Instituto Reuters em abril de 2020 para o Estudo do Jornalismo na Argentina, Estados Unidos, Coréia do Sul, Reino Unido, Alemanha e Espanha ${ }^{16}$, mostrou que cientistas e organizações de saúde nacionais e internacionais foram citados como as fontes de informações mais confiáveis sobre o coronavírus. Organizações de notícias profissionais também estavam relativamente bem posicionadas, ao contrário da classe política e das redes sociais.

Em outro estudo realizado na Suécia, ao serem questionados sobre a confiança em cada um dos grupos de profissionais ou entidades apresentados um uma lista, quando comentam sobre o coronavírus na mídia local, $91 \%$ do público pesquisado diziam confiar muito em declarações dadas por médicos e profissionais de saúde na mídia, $87 \%$ em pesquisadores, $60 \%$ no governo, $30 \%$ nos políticos e apenas $17 \%$ nos jornalistas ${ }^{17}$. Trabalho similar foi realizado na Itália, pelo Observa Science in Society Monitor ${ }^{18}$. Em survey que entrevistou 1.029 pessoas com idade acima de 15 anos (amostra representativa da população nacional por sexo, idade e área de residência), entre 2 e 9 de abril, a maior parte dos entrevistados $(48,4 \%)$ dizia confiar em instituições nacionais e locais para indicações sobre como evitar o contágio. Emissoras de TV e rádio apareceram em segundo lugar $(22,4 \%)$, seguidas do médico que consulta habitualmente $(13,5 \%)$. Com relação à busca de informação sobre COVID-19, 66,6\% diziam recorrer à televisão e ao rádio, $14,7 \%$ a sites institucionais (como do Ministério da Saúde), $11,2 \%$ aos jornais diários e $4,2 \%$ aos contatos em redes sociais.

No Brasil, segundo pesquisa realizada pelo Ibope Inteligência ${ }^{19}$ entre os dias 20 e 23 de março de 2020, 93\% dos entrevistados se diziam preocupados com a COVID-19 e 37\% tinham certeza de que ele(a) ou algum de seus familiares e 
amigos teriam a doença. Entre os entrevistados, $97 \%$ afirmavam que lavam as mãos com frequência, 91\% evitam locais com muitas pessoas, $85 \%$ evitam beijar ou dar a mão ao cumprimentar alguém e $84 \%$ estão saindo de casa com menos fre-

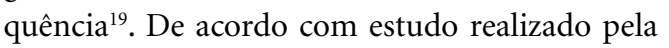
Qualibest ${ }^{20}$, a proporção dos que estão com muito medo do coronavírus cresceu nos primeiros meses da pandemia. Em estudo realizado em dez países, entre os dias 6 e 10 de março de 2020, sete em cada dez entrevistados afirmavam seguir notícias sobre o coronavírus diariamente e, no Brasil, 85\% diziam temer que haja muitas fake news e se preocupar com notícias falsas sobre o vírus. Ainda, 52\% afirmavam estar sendo difícil encontrar informações de credibilidade e confiáveis sobre a doença e $89 \%$ afirmavam que devemos ouvir mais os cientistas e menos os políticos ${ }^{21}$.

Tais estudos de percepção, entendimento e adesão às recomendações de autoridades sanitárias, em seu conjunto, com especial atenção ao período de realização e aos diferentes contextos diante da pandemia, colaboram com a construção de um corpo sólido de conhecimento sobre possibilidades de enfrentamento da COVID-19 e outras possíveis pandemias futuras que dependem da adesão da população.

\section{Metodologia}

Este estudo foi realizado no contexto de uma pesquisa mais ampla, sobre como brasileiros e brasileiras veem a Fundação Oswaldo Cruz (Fiocruz), instituição de pesquisa em saúde cuja missão é "promover a saúde e o desenvolvimento social, gerar e difundir conhecimento científico e tecnológico e ser um agente da cidadania", marcando a passagem de seus 120 anos. Tendo em vista a situação de pandemia que tem assolado o país, optou-se por incluir questões relacionadas à COVID-19, como será detalhado a seguir.

Utilizou-se a técnica de survey, aplicada por meio telefônico (Computer Assisted Telephone Interviewing - CATI), em plataforma online. Optouse por investigar a percepção de pessoas residentes em cidades brasileiras onde a Fiocruz possui unidades técnico-científicas, escritórios técnicos e/ ou projeto especial, a saber: Belo Horizonte-MG, Brasília-DF, Campo Grande-MS, Curitiba-PR, Eusébio-CE, Manaus-AM, Petrópolis-RJ, Porto Velho-RO, Recife-PE, Rio de Janeiro-RJ, Salvador -BA e Teresina-PI. Devido a essa decisão metodológica, explicada pelo contexto da pesquisa mais ampla do qual essa análise sobre a percepção a respeito da COVID-19 faz parte, alguns centros urbanos importantes, como a cidade de São Paulo-SP, não foram incluídos na investigação.

A aplicação dos questionários foi realizada pela empresa Polis Pesquisa. Foram realizados 15 pré-testes, na plataforma de aplicação da entrevista, para ajustes de formulação e de escalas. $\mathrm{O}$ tempo médio de aplicação do questionário completo foi de 33 minutos.

Foram realizadas 1.724 entrevistas, dentre as quais 1.643 entrevistados com idade acima de 16 anos conhecem a Fiocruz e/ou uma de suas unidades, mesmo que superficialmente ("ouvir falar”). Apenas esses entrevistados responderam ao questionário completo. A seleção do morador do domicílio nas cidades pesquisadas foi controlada pelo preenchimento de cotas por sexo e idade, segundo os parâmetros populacionais do Censo Demográfico do Brasil de 2010 do Instituto Brasileiro de Geografia e Estatística (IBGE), sendo que o nível de confiança do estudo é de 95\% e a margem de erro máxima estimada, considerando um modelo de amostragem aleatória simples, é de 2,8 pontos percentuais para mais ou para menos sobre os resultados encontrados no total da amostra. Embora os resultados não possam ser generalizados para todo o país - mas, sim, para as cidades em que o estudo foi realizado -, argumentamos a importância da realização deste estudo em cidades em que há a presença de uma instituição de pesquisa em saúde do peso da Fiocruz, em um contexto de pandemia.

As entrevistas foram aplicadas entre os dias 18 de maio e 10 de junho de 2020. No último dia da coleta, o número de casos confirmados no Brasil era 772.416 e de mortes 39.680. Em 24 de maio de 2021, quando este artigo estava em finalização, havia 16.083.258 casos confirmados e 449.068 mortes, com taxa de letalidade de 2,8\%, taxa de mortalidade de 213,7 por 100.000 habitantes e taxa de incidência de $7.653,3$ por 100.000 habitantes $^{22}$. O questionário foi composto por blocos abordando assuntos relacionados à atuação da Fiocruz, à saúde, à vacinação e às percepções sobre a COVID-19. No total, foram incluídas 18 perguntas sobre a COVID-19, foco deste artigo.

\section{Resultados e discussão}

\section{Percepção da gravidade e perigos da COVID-19}

A maioria dos entrevistados percebe a gravidade da pandemia de COVID-19, concordando 
totalmente ou em parte com a afirmação de que “a pandemia está fora de controle e é uma ameaça a todos nós” (55\% e $27 \%$, respectivamente) e discordando totalmente ou em parte da frase "a COVID-19 tem o mesmo perigo de uma gripe comum" (67\% e 13\%, respectivamente), como mostra o Gráfico 1. No entanto, metade dos entrevistados concorda, pelo menos parcialmente, que ocorrem exageros relacionados aos perigos da doença que circulam no ambiente informacional. Os entrevistados nas faixas etárias acima de 45 anos, com Ensino Médio, renda domiciliar de até dois salários mínimos e evangélicos apresentam maior concordância com a frase "Tem muito exagero sobre os perigos da COVID-19" e os entrevistados na faixa etária entre 18 e 34 anos com ensino universitário, renda domiciliar entre cinco e dez salários mínimos, agnósticos, ateus e os sem religião apresentam maior discordância da afirmação.

Contudo, em uma outra afirmação semelhante, ao apontar para o sujeito da ação - "os cientistas estão exagerando sobre os perigos da COVID-19" - a percepção se altera radicalmente: $72,6 \%$ discordam parcial ou totalmente, apenas $9 \%$ concordam totalmente e $17,7 \%$ concordam parcialmente com a afirmativa, sendo que entre homens, idade acima de 45 anos, com Ensino Fundamental, renda domiciliar de um a dois salários mínimos e evangélicos e cristãos há maior incidência de concordância. Junto aos entrevistados na faixa de 18 a 24 anos e com ensino superior, a incidência de discordância é maior.

Os dados estão em sintonia com estudos nacionais e internacionais, nos quais se verifica que a maior parte da população das localidades pesquisadas percebe o risco e se preocupa com a COVID-19, considerando os altos percentuais de medo de contrair a doença (individual ou de familiares) ou por acreditar que em algum momento poderá ocorrer o contágio ${ }^{15,19,20,23,24,25}$. No Brasil e na Suécia, grande parte dos entrevistados diz se preocupar com notícias alarmistas que aumentam o tamanho do problema ou fake news $^{17,20,21}$.

Chama atenção a confiança depositada nos cientistas. Apesar de 51\% dos entrevistados concordarem totalmente ou em parte (22\% e $29 \%)$ de que há muito exagero sobre os perigos da $\mathrm{CO}$ VID-19, somente $27 \%$ atribuem aos cientistas tais exageros (concordam totalmente, 9\%, ou em parte, 18\%). Isso demonstra que a confiança na expertise continua alta, como veremos a seguir.

\section{Confiabilidade e fontes de informações}

Em nossa survey, quatro são as fontes apontadas como as mais confiáveis: médicos e profissionais da saúde; Organização Mundial da Saúde (OMS); cientistas de universidades ou institutos de pesquisa; e Ministério da Saúde (Gráfico 2).

Escolaridade e renda domiciliar são as variáveis que mantêm uma relação de associação estatisticamente significante com a escolha de "em quem acreditar”, ou seja, em relação àqueles em quem nutrem confiança. Aqueles com escolaridade universitária e pós-graduação tendem a expressar maior confiança na OMS do que as faixas inferiores de escolaridade; já aqueles com escolaridade até o Ensino Médio tendem a manifestar

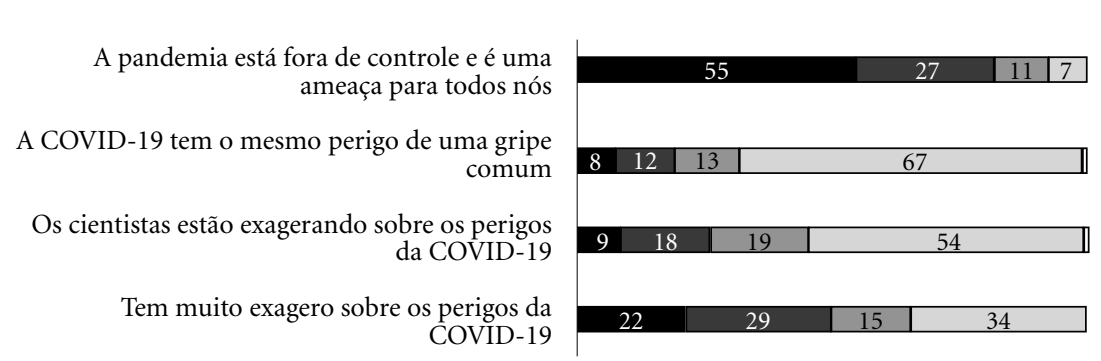

Concordo totalmente $\square$ Concordo em parte $\square$ Discordo em parte $\square$ Discordo totalmente $\square$ Não sei dizer

Gráfico 1. Concordância com frases sobre perigos da pandemia.

Nota: P7.1 - P7.8. "Vou ler oito frases sobre a COVID-19 para que você me diga o quanto você concorda com cada uma delas". Base: 1.643 respostas.

Fonte: Elaborado pelos autores. 


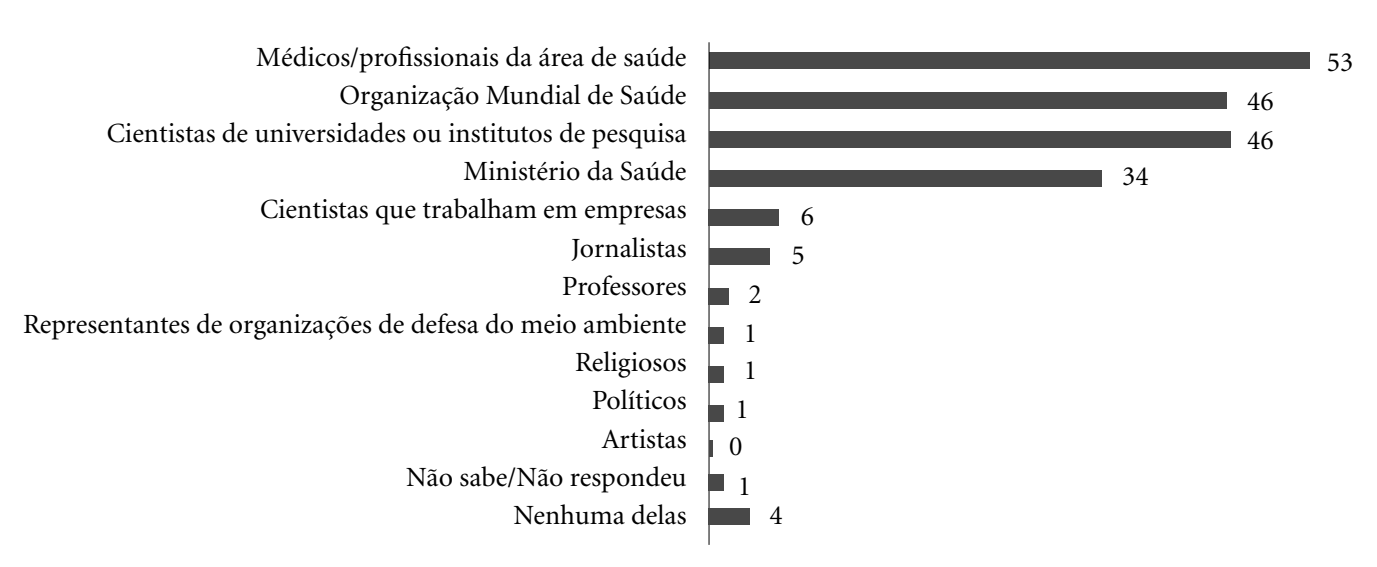

Gráfico 2. Fontes confiáveis de informação sobre a COVID-19.

Nota: P3 e P4. "Dessas fontes que dão informação sobre a COVID-19 que vou ler para você, qual delas você mais confia?", "E qual destas que dão informação sobre a COVID-19 é a segunda que você mais confia?". Base: 1.643 entrevistados.

Fonte: Elaborado pelos autores.

mais confiança no Ministério da Saúde do que nas categorias de formação universitária e pósgraduação. No Ensino Fundamental, há maior frequência de confiança em igrejas e religiosos do que nas demais categorias de escolaridade. E na formação universitária há maior manifestação de confiança em jornalistas do que nas demais faixas.

Entre os entrevistados, $80 \%$ procuram informações ativamente sobre a doença, sem diferenças significativas por gênero, faixa etária, renda domiciliar e raça. Entretanto, a escolaridade formal apresenta algum poder preditivo sobre esse comportamento: quanto maior a escolaridade formal, maior tende a ser a frequência na busca ativa por informações relacionadas à COVID-19. As informações são obtidas de múltiplas origens, com destaque para a televisão (74\%), sites oficiais da área de saúde (69\%), os médicos (59\%) e os jornais impressos ou online (55\%) (Gráfico 3).

Em interações múltiplas, interpessoais e virtuais, os entrevistados da amostra também trocam informações com amigos (51\%), seja comentando informações com as quais interagiram ou consumindo informações por eles obtidas. Os respondentes obtêm informações das mídias sociais (49\%), de pesquisadores (48\%), parentes (45\%), WhatsApp (29\%), rádio (25\%) e, por fim, as igrejas e instituições religiosas aparecem como fonte de informação para apenas $8 \%$.

Com a circulação frequente de informações nem sempre verdadeiras sobre a doença e sobre a melhor forma de enfrentá-la, uma expressiva maioria manifesta desconfiança sobre as notícias que recebe: $35 \%$ concordam totalmente e $35 \%$ em parte com a frase "quando recebo informações sobre a COVID-19 a minha primeira reação é desconfiar". Mesmo com a grande complexidade do ambiente informacional e o fato de os indivíduos, em geral, buscarem ativamente informações sobre a COVID-19, a maior confiança dos entrevistados da amostra é dirigida às fontes oficiais e capacitadas - tanto internacionais quanto nacionais e também profissionais especializados -, que agregam pesquisa, conhecimento e credibilidade, como visto acima.

Além da alta percepção de risco de contágio e das precauções adotadas, outros estudos também apontam que os entrevistados se declaram bem-informados sobre a pandemia buscando informações com frequência alta, sendo as principais fontes TV (aberta ou paga), sites ou portais de notícias e redes sociais ${ }^{17,19,20}$. Quando questionados sobre os porta-vozes mais confiáveis para serem ouvidos sobre a pandemia, temos uma informação muito positiva: os cientistas e as autoridades sanitárias ganham destaque no Brasil, na Alemanha e na Suécia e são considerados como os mais confiáveis ${ }^{15,17,21}$.

A confiabilidade dos veículos de comunicação, segundo o Ibope inteligência ${ }^{19}$ no entanto, é preocupante quando focamos nas redes sociais: $69 \%$ as consideram confiáveis. Em contrapartida, a maior parte dos entrevistados brasileiros (89\%) no estudo realizado pela Edelman Trust Baro- 


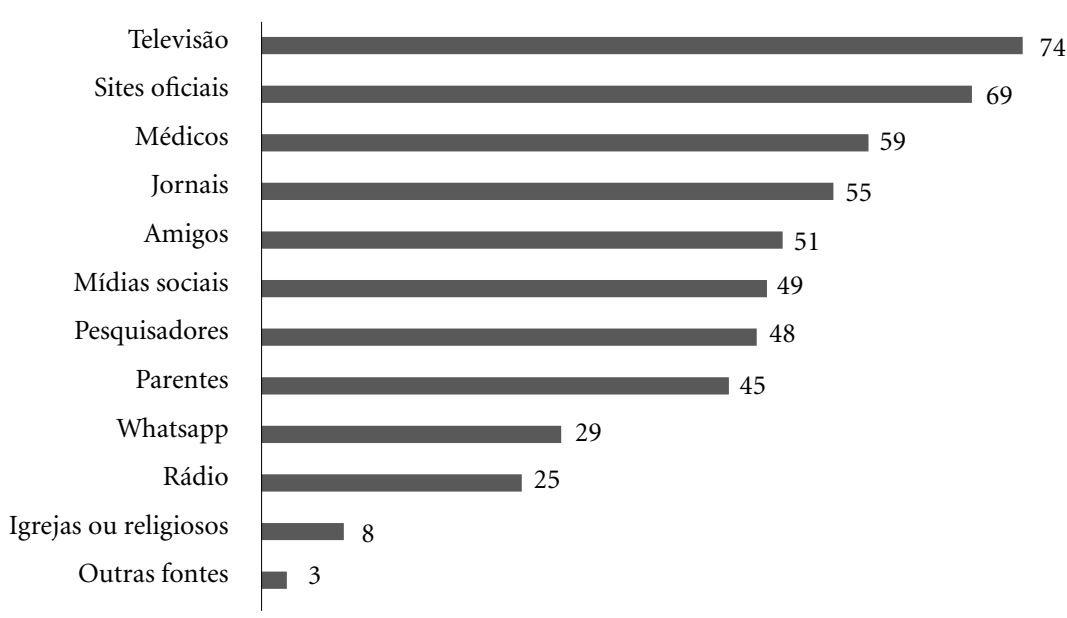

Gráfico 3. Onde buscam informação sobre COVID-19.

Nota: P2. "Eu vou citar algumas fontes de informação para que você me diga onde você obtém informação sobre a COVID-19. Você obtém informação sobre a COVID-19 na(o)...”. Base: 1.306 entrevistados (procuram ativamente informações sobre a COVID-19).

Fonte: Elaborado pelos autores.

meter ${ }^{21}$ concorda que "deveríamos ouvir mais os cientistas e menos os políticos" e $58 \%$ se dizem preocupados com a politização da crise sanitária.

\section{Checagem de informações sobre a COVID-19}

Enquanto oito em cada dez respondentes informam o hábito de checar informações que recebem sobre a COVID-19, dois em cada dez fazem-no às vezes ou admitem não conferir a veracidade da informação que recebem. $\mathrm{O}$ hábito de checar as informações está mais presente entre jovens da faixa de 18 a 24 anos e, em tendência inversa, na faixa etária dos mais velhos, acima de 55 anos, é mais incidente o hábito de não verificar as informações em relação às demais categorias etárias. A escolaridade é um bom preditor para a presença do hábito de checar a informação recebida: nas faixas de alta escolaridade - no nível universitário e principalmente na pós-graduação - são mais frequentes os relatos de verificação do que nas categorias de escolaridade Fundamental e do Ensino Médio.

Neste momento de pandemia e de grande alerta social para os riscos da doença, ganham em credibilidade as instituições consolidadas, principalmente as instituições de pesquisa científica e órgãos governamentais e internacionais de saúde, as instituições de imprensa e os médicos e profissionais da área de saúde. Entre as fontes buscadas por aqueles que têm o hábito de checar as informações relacionadas a eventual novo tratamento ou medicamento para a COVID-19, é grande a procura virtual $(66 \%)$ para os sites institucionais, principalmente vinculados à pesquisa científica no campo da saúde, assim como em sites de órgãos governamentais, como o do Ministério da Saúde, seguidos da imprensa profissional (18\%), médicos e profissionais da área de saúde e publicações e artigos médico-científi$\cos (10 \%)$. Poucos afirmam buscar a verificação de informações nas redes sociais (4\%) ou com amigos e familiares (1\%).

\section{Atitudes, precauções e prioridades para o enfrentamento}

A maior parte dos entrevistados concorda (totalmente + parcialmente) que se seguir as orientações e precauções indicadas principalmente por instituições nacionais e internacionais de saúde para evitar o risco de contágio não corre o risco de ser contaminados pelo Sars-CoV-2 (vírus causador da COVID-19) e que a única forma de evitar o contágio é ficar em casa $(82 \%$ e $77 \%$, respectivamente). Os jovens com idade entre 18 e 24 anos são os que mais discordam da frase "Se eu seguir as precauções que estão sendo indicadas eu não corro o risco de ser contaminado" e 
os que mais concordam são os com 55 anos ou mais. As mulheres apresentam maior frequência de concordância total com a afirmação "A única forma de evitar o contágio é ficar em casa" do que os homens, assim como os entrevistados com 55 anos ou mais e os com escolaridade mais baixa. Os que mais discordam dessa frase são os que vivem em domicílios com renda acima de 20 salários mínimos.

A maioria dos entrevistados discorda pelo menos parcialmente de que as pessoas não podem fazer nada para evitar o contágio da COVID-19. Embora concordem com as recomendações indicadas pelos cientistas, profissionais da saúde e instituições da saúde (79\%), nas camadas de baixa renda domiciliar (até dois salários mínimos) há maior proporção do que nas demais faixas de renda de concordância de impossibilidade de segui-las por falta de recursos. Nas faixas de renda domiciliar de cinco a vinte salários mínimos há maior proporção de concordância e aderência às recomendações; na faixa mais alta de renda domiciliar há maior inclinação pela discordância e não aderência em relação às demais categorias de renda domiciliar (Gráfico 4).

Além das diferenças por renda domiciliar, as mulheres tendem a concordar mais do que os homens com as recomendações; homens tendem mais a justificar a falta de recursos do que mulheres para evitá-las; homens também discordam e rejeitam mais do que as mulheres as recomendações para o enfrentamento da COVID-19. Enquanto na escolaridade superior - ensino superior e pós-graduação - há maior incidência de concordâncias e aderência às orientações do que nas demais faixas de escolaridade, na escolaridade Fundamental até o quinto ano e na faixa do Ensino Médio há maior frequência de concordância, mas impossibilidade de seguir as orientações.

A percepção de risco de contágio se reflete em ações individuais de autoproteção em outros estudos publicados, sendo que grande parte dos entrevistados declara lavar as mãos com frequência, evitar lugares com muitas pessoas, evitar contato físico, sair de casa com menor frequência ou em horários alternativos, entre outros ${ }^{15,19,24,25}$. Cabe ressaltar que se verifica uma tendência de mudança de adesão às recomendações das autoridades sanitárias no decorrer da linha do tempo da pandemia, relacionada ao apoio à volta ao trabalho das pessoas que não fazem parte do grupo de risco, proporção dos que são favoráveis ao isolamento social para todas as pessoas, inde-
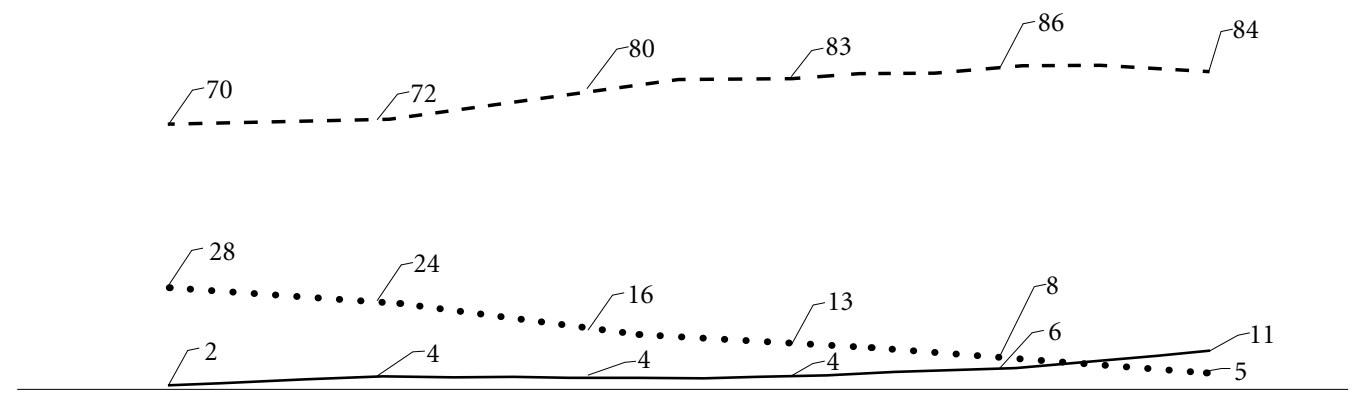

Até 1 salário Acima de 1 até 2 Acima de 2 até 5 Acima de 5 até 10 Acima de 10 até 20 Acima de 20 mínimo salários mínimos salários mínimos salários mínimos salários mínimos salários mínimos

$\begin{array}{ll}- \text { - }^{-} & \text {Eu concordo com as recomendações e estou seguindo } \\ \cdots & \text { Eu concordo com as recomendações, mas não tenho recursos para seguir } \\ & \text { Eu discordo das recomendações que estão sendo dadas e não estou seguindo }\end{array}$

Gráfico 4. Aderência às recomendações por faixa de renda.

Nota: Base: 1.643 entrevistados.

Fonte: Elaborado pelos autores. 
pendentemente de ser ou não grupo de risco e pessoas que estão tomando cuidado, mas saindo para trabalhar ou outras atividades ${ }^{24,25}$.

Nesse contexto, para 63\% dos entrevistados do estudo apresentado, o enfrentamento à emergência da COVID-19 deve ser feito principalmente em ações integradas, focadas na redução da circulação, no investimento de recursos financeiros para desenvolver a vacina e medicamentos e também na ajuda financeira às empresas e às pessoas.

\section{A ciência e a COVID-19}

Neste momento de pandemia, os entrevistados depositam grande confiança nos cientistas: $97,3 \%$ acreditam que a ciência encontrará a cura. Esses que demonstram confiança, contudo, dividem-se quanto ao timing em que a solução será apresentada: 45\% acreditam que será "logo" e $52 \%$ consideram que vai demorar. Apenas 3\% dizem não acreditar que os cientistas encontrarão uma solução para a COVID-19 (Gráfico 5).

Em relação à capacidade de comunicação e clareza das informações concedidas pelos cientistas em entrevistas, blogs, vídeos ou sites sobre a COVID-19, 42\% consideram-nas claras e efetivas; $26 \%$ acham que as informações prestadas pelos cientistas são conflitantes e estão confundindo as pessoas; $16 \%$ consideram que os cientistas não deveriam falar em público, mantendo as suas opiniões restritas às instituições; e 16\% acreditam que os cientistas sejam muito competentes, mas não sabem se comunicar.
Diante desse desafio de dimensões globais e da crise provocada pelo impacto da doença, a comunidade científica está mobilizada em busca de respostas e mitigação da pandemia, buscando soluções para um problema já instalado e que foi apontado mesmo que de forma indireta em análises de risco anteriores. O princípio da precaução ${ }^{9}$ não foi acionado e análises de risco conflitantes apresentadas pela comunidade científica contribuíram para o adiamento de medidas que poderiam ter minimizado ou evitado a pandemia. A dificuldade da ciência em explicitar provas absolutas com estimativas que por vezes diferem entre si e, muitas vezes, com estudos pontuais sem uma visão ampla e conectada geram desconfiança e dificuldade de engajamento da sociedade civil. Nesse sentido, Hannigan ${ }^{9}$ comenta sobre questões ambientais controversas:

O que particularmente abre as portas para a criação e contestação de problemas ambientais é a incapacidade da ciência de fornecer provas absolutas - evidências inequívocas de segurança. Em vez disso, os cientistas oferecem estimativas de probabilidade que geralmente variam amplamente de um para outro ${ }^{9}$ (p.97).

A ciência, muitas vezes, é controversa e dependente de narrativas mais ou menos aderentes ao contexto e aos interesses que estão em jogo ${ }^{9-}$ ${ }^{11}$, apresentando análises técnicas nem sempre compreensíveis para grande parte da população, utilizando enquadramentos e narrativas que não estabelecem diálogos efetivos que considerem particularidades ou busquem uma construção conjunta. Além das dificuldades por parte das au-

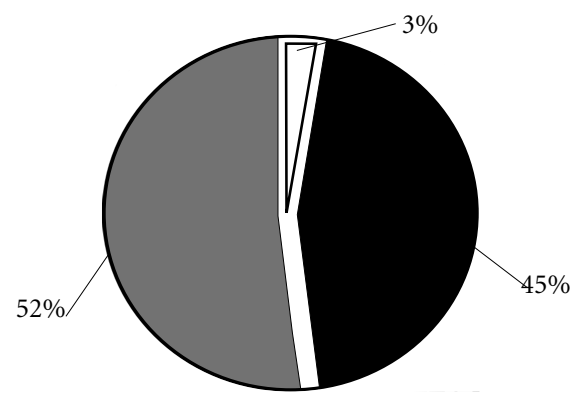

Não acredito que os cientistas irão encontrar uma solução para a Covid-19

Acredito que os cientistas logo encontrarão uma forma de conter a Covid-19

Acredito que os cientistas irão encontrar uma solução, mas vai demorar

Gráfico 5. Confiança na ciência e nos cientistas.

Nota: P9. “Pensando na ciência e nos cientistas, com qual dessas frases mais concorda?”. Base: 1.643 entrevistados. 
toridades epistêmicas, os meios de comunicação também constroem suas narrativas e concorrem com informações disseminadas no meio digital, onde as mídias sociais têm grande impacto. $\mathrm{Na}$ internet e nas redes sociais, opiniões contra ou a favor sobre variados temas são lidas e debatidas. A avalanche de fake news sobre os mais variados temas, incluindo a COVID-19, com narrativas de grande apelo, faz com que diferentes públicos adotem e compartilhem informações (ou desinformações) sem a preocupação com a veracidade ou embasamento do conteúdo, gerando mais controvérsias e atitudes conflitantes.

\section{Comentários finais}

Os resultados apresentados neste artigo, mesmo abrangendo cidades onde a Fiocruz está fisicamente presente, fornecem informações que propiciam ações mais assertivas e indicam a necessidade de estudos em outras localidades e triangulação de dados que abordem o tema em grupos sociais específicos para o entendimento mais aprofundado de diferentes realidades, considerando particularidades temporais da doença e construindo diálogos eficazes para o enfrentamento da COVID-19 e desafios futuros. É necessário considerar condições socioeconômicas, políticas e culturais, formas de enfrentamento adotadas pelas autoridades locais, reais condições de adoção de medidas, além de especial atenção à linha do tempo da doença (nível de contágio, mortes e novas descobertas) em cada localidade, configurando um cenário de várias pandemias concomitantes de uma mesma doença.

A pandemia da COVID-19 traz desafios emergenciais que apontam a necessidade de um novo olhar para a comunicação da ciência e para relação entre ciência e sociedade, ultrapassando os limites das análises técnicas de riscos. No cenário atual, com o senso de urgência e a potencialização e vivência das incertezas acerca do presente e do futuro, a necessidade de alinhamento da comunicação e o fortalecimento da confiança nas instituições se fazem presentes. Para enfrentar e mitigar a pandemia, se faz necessário um olhar atento para o engajamento consistente e sustentável da população em diferentes contextos socioeconômicos, políticos e culturais.

As pesquisas de percepção são de grande valor para a construção de pontes de conexão efetivas, tanto do ponto de vista da comunicação como da definição de ações e de diálogos construtivos e mais aderentes a diferentes públicos, trazendo os diversos conflitos, perspectivas e caminhos possíveis para contribuir para o fortalecimento do campo da saúde global e a busca de modos de vida sustentáveis. Apesar de certo senso comum que imagina que a desinformação já tenha abalado a confiança das pessoas em fontes oficiais de informação e em cientistas, nossos dados mostram que há excelente potencial para ações que saibam escutar os públicos, levando em consideração suas expectativas e percepções.

\section{Colaboradores}

Todos os autores (exceto B Maakaroun) contribuíram para a concepção e o desenho do estudo. L Massarani e IM Mendes contribuíram com a análise e a interpretação dos dados, a concepção e o delineamento do artigo e a redação do mesmo. C Polino, Y Castelfranchi e V Fagundes contribuíram com análise e redação. B Maakaroun contribuiu com a coleta, a consolidação e a análise. Todos os autores revisaram e aprovaram a redação final. 


\section{Agradecimentos}

Agradecemos ao Instituto Nacional de Comunicação Pública da Ciência e Tecnologia (INCTCPCT), no âmbito do qual este estudo foi realizado, e à Fundação Oswaldo Cruz, pelo apoio a este estudo. Além disso, agradecemos ao CNPq e à Faperj, pelo apoio ao INCT-CPCT. L Massarani agradece o apoio do CNPq pela Bolsa Produtividade $1 \mathrm{~B}$ e da Faperj pela bolsa Cientista do Nosso Estado. Agradecemos a Carlos Gadelha, Paulo Roberto Elian dos Santos, Maria Ines Rodrigues e Karla Bernardo Mattoso Montenegro na consolidação deste estudo.

\section{Referências}

1. Morens DM, Folkers GK, Fauci AS. O desafio das doenças infecciosas emergentes e reemergentes. Natureza 2004; 430:242-249.

2. Hirschfeld K. Microbial insurgency: Theorizing global health in the Anthropocene. Antropocene Rev 2019; 7(1):3-18.

3. Wilkinson A. Local response in health emergencies: key considerations for addressing the COVID-19 pandemic in informal urban settlements. Londres: International Institute for Environment and Development; 2020.

4. Bambra C, Riordan R, Ford J, Mattews F. The COVID-19 pandemic and health inequalities. J Epidemiol Community Health 2020; 74(11):964-968.

5. Baqui P, Bica J, Marra V, Ercole A, Schaar M. Ethnic and regional variations in hospital mortality from COVID-19 in Brazil: a cross-sectional observational study. Lancet Glob Health 2020; 8:e1018-e1026.

6. Garcia PJ, Alarco A, Bayer A, Buss P, Guerra G, Ribeiro H, Rojas K, Saenz R, Snyder NS, Solimano G, Torres R, Tobar S, Tuesca R, Vargas G, Atun R. COVID-19 Response in Latin America. Am J Trop Med Hyg 2020; 103(5):1765-1772.

7. Di Giulio GM, Vasconcellos MP, Gunther WMR, Ribeiro H, Assunção JV. Percepção de risco: um campo de interesse para a interface ambiente, saúde e sustentabilidade. Saude Soc 2015; 24(4):1217-1231.

8. Organização Mundial de Saúde (OMS). Comunicação de riscos em emergências de saúde pública: um guia da OMS para políticas e práticas em comunicação de risco de emergência. Genebra: OMS; 2018.

9. Hannigan J. Environmental sociology. Londres: Taylor \& Francis e-Library; 2006

10. Leite JC. Controvérsias na climatologia: o IPCC e o aquecimento global antropogênico. Scientice Studia 2015; 13(3):643-677.

11. Viglio JE, Di Giulio GM, Barbi F, Ferreira LC. Narrativas científicas sobre petróleo e mudanças do clima e suas reverberações na política climática brasileira. Sociologias 2019; 21(51):124-158.

12. Moreno $\mathrm{AB}$, Coeli $\mathrm{CM}$, Munk S. Informação em saúde. Dicionário da educação profissional em saúde [Internet]. 2009 [acessado 2021 mar 01]. Disponível em: http://www.sites.epsjv.fiocruz.br/dicionario/verbetes/ infsau.html.

13. Wardle C, Derakhshan H. Information disorder: toward an interdisciplinary framework for research and policy making. Estrasburgo: Council of Europe Report; 2017.

14. Organização Pan-Americana de Saúde (OPAS). Folha informativa COVID-19 - Escritório da OPAS e da OMS no Brasil [Internet]. 2020 [acessado 2020 set 22]. Disponível em: https://www.paho.org/pt/covid19.

15. Gerhold L. COVID-19: Risk perception and coping strategies. Results from a survey in Germany [Internet]. 2020 [acessado 2020 set 22]. Disponível em: https:// psyarxiv.com/xmpk4/.

16. Brennen JS, Simon F, Howard P, Nielsen R. Types, sources, and claims of COVID-19 misinformation. Oxford: Reuters Institute for the Study of Journalism; 2020. 
17. Karolinska Institute and Södertörn University, va (public \& science/kantar sifo). Coronavirus in the Swedish media study - high public confidence in researchers and healthcare professionals [Internet]. 2020 [acessado 2020 out 22]. Disponível em: https://v-a.se/2020/04/ coronavirus-in-the-swedish-media-study-high-public-confidence-in-researchers-and-healthcare-professionals/.

18. Observa Science in Society Monitor. Survey Italian Citizens and Covid-19: one month later - April 2020 [Internet]. 2020 [acessado 2020 out 22]. Disponível em https://sagepus.blogspot.com/2020/04/italian-citizens-and-covid-19-one-month.html.

19. Ibope Inteligência. Covid-19: percepção, opinião, comportamento e hábitos de consumo [Internet]. 2020 [acessado 2020 out 22]. Disponível em: https://www. ibopeinteligencia.com/noticias-e-pesquisas/covid19-percepcao-opiniao-comportamento-e-habitosde-consumo/.

20. Qualibest. Comportamento do consumidor após o CORONAVÍRUS - onda 4 [Internet]. 2020 [acessado 2020 jul 21]. Disponível em: https://www.institutoqualibest.com/wp-content/uploads/2020/04/0417-Coronavi\%CC $\% 81$ rus-Mi\%CC $\% 81$ dia-Branding-e-Influenciadores.pdf.

21. Edelman Trust Barometer. Spring Update: Trust and the Covid-19 Pandemic [Internet]. 2020 [acessado 2020 jul 21]. Disponível em: https://www.edelman. com/sites/g/files/aatuss191/files/2020-05/2020\%20 Edelman\%20Trust\%20Barometer\%20Spring\%20 Update.pdf.

22. Conselho Nacional dos Secretários de Saúde (CONASS). Painel CONASS - COVID-19 [Internet]. 2020 [acessado 2021 maio 24]. Disponível em: http://www. conass.org.br/painelconasscovid19/.
23. BTG Pactual. Os brasileiros e o Coronavírus: visões sobre a pandemia e seus impactos econômicos [Internet]. 2020 [acessado 2021 maio 24]. Disponível em: https://www.btgpactualdigital.com/blog/coronavirus/ pesquisa-os-brasileiros-e-o-coronavirus\#: : :text=Veja $\% 20$ mais $\% 20$ alguns $\% 20$ dados $\% 20$ coletados,pode $\% 20$ ter $\% 20$ consequ $\%$ C3\%AAncias $\% 20$ sanit $\%$ C3\%Alrias\%20graves.

24. DATAFOLHA. Opinião sobre isolamento social e lockdown [Internet]. 2020 [acessado 2020 set 13]. Disponível em: http://media.folha.uol.com.br/datafolha/2020/05/27/5aabciel8238c225b8a943e4b6ed8a9802pand5.pdf.

25. DATAFOLHA. Isolamento social. Medo de ser contaminado [Internet]. 2020 [acessado 2020 out 13]. Disponível em: http://media.folha.uol.com.br/datafolha/2020/08/19/34c9d0ae243b593db19bc9652df8d054reab.pdf.

Artigo apresentado em 15/12/2020

Aprovado em 01/06/2021

Versão final apresentada em 03/06/2021

Editores-chefes: Romeu Gomes, Antônio Augusto Moura da Silva 